\title{
Thrombotic thrombocytopenic purpura specialist centres: providing better outcomes for patients with rare but life- threatening conditions
}

\author{
Authors: Tina Dutt, Vanessa Martlew, Cheng Hock Toh and Clare Kay Jones
}

\section{Aims}

To demonstrate the value of TTP specialist centres in improving outcomes for patients with TTP.

\section{Background}

Specialist centres for stroke and acute coronary events improve patient survival. Similarly, the best model of care for reducing morbidity and mortality for patients with complex, rare diseases should be delivered by a dedicated, specialist team.

Thrombotic thrombocytopenic purpura (TTP) is a medical emergency requiring urgent multispecialty input at the point of presentation. Patients develop widespread thromboses and profound thrombocytopenia. Undetected / left untreated, the condition is usually fatal, with $90 \%$ mortality.

Plasma exchange is the only treatment shown to reduce the mortality of TTP. National guidelines advise that patients should receive treatment within 4-8 hours of presentation.

To achieve this, TTP needs to be promptly diagnosed, followed by effective coordination of multispecialty input to facilitate life-saving treatment.

\section{Methods}

An internal review of the governance framework to manage patients presenting/referred to our tertiary referral hospital with TTP within was performed in 2012. It was found that patients across the region were experiencing significant delays in diagnosis and treatment, resulting in death. Four of nine patients ultimately managed at our trust in 12 months died. Time to diagnosis ranged from 3 hours to 3 weeks, and time to plasma exchange from referral from 5 hours to 3 days.

To improve practice and service delivery for this vulnerable patient group, our team embarked upon acute service redesign to provide a regional, specialist service for TTP.

\section{Results}

A number of changes were implemented over a 6-month period. Formalised care pathways were developed, with the target that patients receive plasma exchange, a service provided by
NHS Blood and Transplant, within 4-8 hours of presentation. Abnormal/characteristic blood films for TTP have been red flagged and urgently reported to the consultant on call. All suspected TTP patients across the region have been blue lighted to our trust for assessment in resus, followed by transfer to a critical care bed and daily specialist nurse input.

Tangible improvements in efficiency have been demonstrated by improved patient outcomes: zero deaths from 15 patients' episodes over 10 months, and average time to plasma exchange: 4 hours.

\section{Conclusions}

Engagement with different specialty teams has allowed us to provide a $24 / 7$ specialist service, ensuring that patients with TTP receive the best care regardless of time or place of presentation. Specialist centres for TTP facilitate coordination of acute care and centralisation of intensive resource and experience, leading to improved patient outcomes.

\section{Conflict of interest statement}

Nil. 